\title{
Observations on the loss of catecholamine fluorescence from intrauterine adrenergic nerves during pregnancy in the guinea-pig
}

\author{
C. Bell and S. J. Malcolm \\ Department of Physiology, University of Melbourne, Parkville, Victoria 3052, Australia
}

\begin{abstract}
Summary. During unilateral pregnancy in the guinea-pig there is loss of formaldehydeinduced fluorescence from the adrenergic nerves supplying the uterus and its vasculature. This loss occurs initially near the site of implantation at about Day 20 of gestation and spreads progressively. Implantation of wax pellets containing progesterone into the uterine lumen or the gastrocnemius muscle of virgin guinea-pigs for 7 days produced loss of fluorescence from all local adrenergic nerves. No diminution of fluorescence was seen when pellets containing oestradiol were substituted. Chronic denervation studies showed that the adrenergic axons supplying the uterus and its arteries originated from both the ovarian artery and the pelvic region. Our results suggest that loss of adrenergic fluorescence within the uterus during pregnancy is due to an effect of placental progesterone which is localized to the uterus because the high concentration of progesterone necessary to cause fluorescence loss is not attained in the systemic circulation.
\end{abstract}

\section{Introduction}

In some species there is, during the course of pregnancy, a progressive disappearance of the characteristic formaldehyde-induced fluorescence normally associated with the adrenergic nerves of the uterus and its arterial supply. There is also a fall in the uterine content of noradrenaline. These declines have been observed in the guinea-pig (Sjöberg, 1968), rabbit (Rosengren \& Sjöberg, 1968), man (Nakanishi, McLean, Wood \& Burnstock, 1968) and dog (Ryan, Clark \& Brody, 1974) and Bell (1972) has suggested that they constitute a protective mechanism against feto-placental ischaemia during generalized maternal sympathetic activation.

During unilateral pregnancy in the guinea-pig the disappearance of fiuorescence occurs in both uterine horns, indicating that it is likely to be hormonally mediated rather than being secondary to mechanical compression of the uterine wall by the products of conception (Sjöberg, 1968). By contrast, no diminution of fluorescence of adrenergic nerves in other regions of the body is seen during pregnancy. The male internal genitalia receive their adrenergic innervation from 'short' pelvic neurones (Sjöstrand, 1965) which exhibit marked differences from the 'long' neurones of the sympathetic chain in their characteristics of transmitter turnover (see Bell, 1972). It has been proposed that the pregnancy-induced loss of fluorescence is restricted to the intrauterine axons because these also arise from 'short' adrenergic neurones lying in the pelvic region (Sjöberg, 1968; Owman, Sjöberg \& Sjöstrand, 1974). Such an explanation is, however, in conflict with evidence presented by other workers which suggests that the adrenergic innervation of the guinea-pig uterus arises primarily from 'long' adrenergic neurones and enters the uterus with the ovarian artery (Isaac, Pennefather \& Silva, 1969; Kulkarni, Wakade \& Kirpekar, 1976). In the present study we have re-examined the origins of the intrauterine adrenergic nerves and made some observations on the probable mechanism of loss of fluorescence during pregnancy. 


\section{Materials and Methods}

The animals were from our outbred colony of smooth-haired guinea-pigs in which the females are reproductively active at $300 \mathrm{~g}$ body weight and the length of gestation is between 63 and 70 days (mean 65 days). The animals used were female fetuses removed by hysterotomy between Days 60 and 65 of gestation, virgin females weighing 300-700 g, and primigravid animals in which the right oviduct had been sectioned during a posterior lateral laparotomy under ether anaesthesia at least 3 weeks before mating to ensure unilateral pregnancy. The day on which a vaginal plug was found was taken as Day 1 of pregnancy and the stage of gestation was confirmed at the time of dissection by comparing the fetal crown-rump lengths with those recorded by Draper (1920).

\section{Histochemistry}

Animals were killed by cervical dislocation and exsanguination. Samples less than $2 \mathrm{~mm}$ thick were taken from the uterine horns and body, oviducts, vagina, radial and main parametrial arteries, mesenteric arteries and atria and were placed on numbered paper tags, frozen in liquid propane and freeze dried at $-40^{\circ} \mathrm{C}$ and $0 \cdot 13 \mathrm{~Pa}$ for at least $40 \mathrm{~h}$. They were then incubated at $80^{\circ} \mathrm{C}$ for $60-90 \mathrm{~min}$ with paraformaldehyde (Merck) equilibrated at $65 \%$ humidity to convert tissue noradrenaline to a fluorescent product (Falck \& Owman, 1965). After embedding in paraffin wax (m.p. $54^{\circ} \mathrm{C}$ ), the sections $(8-10 \mu \mathrm{m})$ were examined under a Leitz Orthoplan microscope fitted with an $\mathrm{HBO} 200$ highpressure mercury burner, a BG12 excitation filter and a 510 or $530 \mu \mathrm{m}$ barrier filter.

\section{Denervations}

A midline laparotomy was performed under pentobarbitone sodium anaesthesia $(38 \mathrm{mg} / \mathrm{kg}$ i.p.). To determine the routes by which adrenergic axons entered the uterus, forceps cooled in liquid nitrogen were used to freeze briefly a $2-\mathrm{mm}$ length of the main parametrial artery or the paracervical ganglia lying between the main parametrial artery and the cervix (Bell, 1973). The parametrial artery was frozen proximal to its cervical branches, at the level of junction of the uterine horns or at the level of the oviduct (Text-fig. 1). In other animals, one uterine horn was divided between ligatures just above its junction with the uterine body. At least 7 days was always allowed after operation for degeneration of any damaged axons to occur.

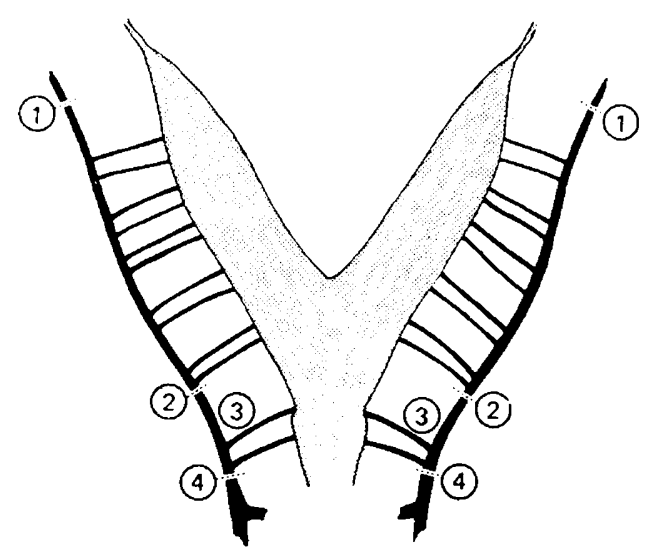

Text-fig. I. Diagrammatic representation of the guinea-pig uterus and its vascular supply. The numbers indicate sites at which freezing was utilized to interrupt axons travelling to the intrauterine blood vessels and myometrium via (1) the ovarian artery or (2) the uterine artery; and to differentiate between those axons reaching the uterine artery via (3) the paracervical ganglia or (4) along the iliac artery. 


\section{Implants}

Elongated pellets weighing $20-50 \mathrm{mg}$ were formed by hand from paraffin wax (m.p. $54^{\circ} \mathrm{C}$, Gurr) containing a suspension of $400 \mathrm{mg}$ progesterone (Sigma)/g or $40 \mathrm{mg}$ oestradiol-17 3 benzoate(Sigma) $/ \mathrm{g}$. For intrauterine placement, a midline laparotomy was performed under pentobarbitone sodium anaesthesia and one pellet containing steroid was inserted into the lumen of the left uterine horn through a transverse incision which was then closed with fine surgical silk. A ligature was placed round the horn below the pellet. A pellet of similar size containing only paraffin wax was inserted into the right uterine horn. For intramuscular placement, a small skin incision was made, under ether anaesthesia, over the lateral portion of the right gastrocnemius muscle and a pellet containing steroid was inserted into a tunnel made by gentle blunt dissection in the belly of the muscle. The skin incision was then closed with surgical silk. A pellet of similar size containing only wax was inserted into the left gastrocnemius muscle.

\section{Perfusions}

Guinea-pigs were killed by cervical dislocation after intracardiac injection of 1000 units heparin sodium. For investigation of intrauterine vascular connections the left uterine artery was cannulated with polythene tubing (PE50) through the common iliac artery. Both ovarian arteries and the contralateral iliac artery above the uterine artery were then ligated. The inferior vena cava was opened and the uterine arterial cannula was connected to a reservoir of modified Krebs solution (McEwan, 1956) with a constant perfusion pressure head of $100 \mathrm{mmHg}$. When all visible blood had been washed from the uterus $0.5 \mathrm{ml}$ India ink (Pelican) was injected into the perfusion stream and the appearance of each uterine horn was observed as the perfusion continued. For investigation of relationships between the uterine radial veins and radial arteries, the main uterine vein was cannulated with polythene (PE50) tubing near the oviduct and ligatures were placed on its cervical end and on the radial veins where they entered the uterine horn. The abdominal aorta was opened and the extrinsic uterine veins were flushed first with $0.9 \%(\mathrm{w} / \mathrm{v}) \mathrm{NaCl}$ solution, then with India ink at a perfusion pressure of $100 \mathrm{~mm} \mathrm{Hg}$. The uterus and its vasculature were then removed in toto from the animal, fixed in $10 \%$ formalin overnight and cleared in glycerol for microscopic inspection by transmitted light.

\section{Results}

\section{Distribution of adrenergic axons in the uterus of the virgin female}

The distribution of adrenergic axons within the uterus was as reported by Sjöberg (1968). The outer longitudinal and inner circular smooth muscle layers contained scattered fluorescent fibres which ran parallel to the direction of the muscle fibres and did not appear to be related to blood vessels. In addition, a dense population of fluorescent fibres was associated with the parametrial arteries and with the small arteries and arterioles running through and between the smooth muscle layers. These axons were closely applied to the outer surface of the arterial media (PI. 1, Fig. 1). No characteristic differences in the pattern or density of innervation were noted along the length of the uterine horns. The appearance of the adrenergic nerves was similar in uteri from animals in oestrus or dioestrus.

\section{Origin of the uterine adrenergic innervation}

Interruption of the nerves reaching the uterus from its ovarian end in 4 animals resulted in degeneration of almost all fluorescent fibres associated with the myometrium and arteries in the ovarian third of the uterine horn. There was a decreased density of fibres in the middle portion of the horn, but the innervation of the cervical third of the horn appeared normal. By contrast, destruction of the paracervical ganglia in 3 animals produced complete or almost complete loss of fluorescence in the cervical third of the horn and loss of some fibres from the middle portion but had no effect on 
fluorescence in the ovarian third. An identical pattern of degeneration was seen in 2 other animals in which the main uterine artery itself was frozen at the level of junction of the uterine horns, but freezing the artery at the level of the cervix had no visible effect on the density of innervation. When the ovarian or uterine artery was denervated the degree of destruction of adrenergic axons associated with particular radial arteries paralleled the degree of destruction occurring within the portion of the horns supplied by these arteries.

Serial sections of the paracervical ganglia in one normal animal revealed that a small number (less than $10 \%$ of the ganglion cells exhibited catecholamine fluorescence. However, serial cross-sections of the vagina and uterine body of 2 animals failed to reveal any fluorescent intramural ganglion cells. In a further 5 animals surgical separation of uterine horns from uterine body had no effect on the density of adrenergic axons in the horns.

\section{Changes during pregnancy in fluorescence of uterine adrenergic axons}

The sequence of loss of uterine fluorescence during a unilateral pregnancy is shown in Text-fig. 2. No change in the pattern or intensity of fluorescence was seen up to Day 16 of gestation. Between Days 16 and 22 there was disappearance of all fluorescence from the uterine wall immediately around the site of implantation. By Day 30 this loss of fluorescence had extended to the face of the pregnant uterine horn opposite the implantation site and to the radial arteries supplying the implantation site. By Day 45 fluorescence was absent from virtually the entire pregnant horn. By Day 55 the area affected had extended to the cervical end of the empty horn and by term fluorescence was absent from both horns and their radial arteries. The typical appearance of the empty horn at the end of gestation is compared with the pattern of fluorescence in a virgin animal in PI. 1, Figs 1 and 2. At no stage of pregnancy did the loss of fluorescence in the radial arteries extend into the main parametrial artery, nor was there any diminution of fluorescence in the adrenergic nerves of the vagina, the oviducts, the mesenteric arteries or the right atrium.

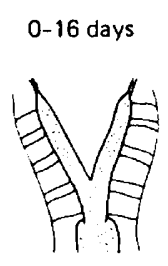

45 days

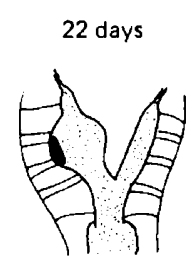

55 days
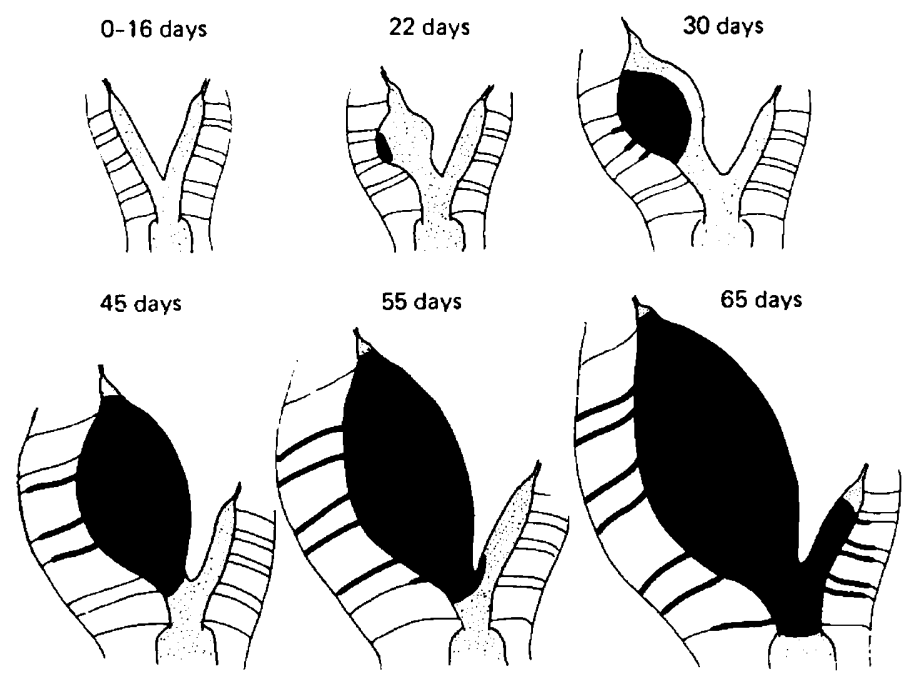

Text-fig. 2. Diagrammatic representation of the progressive loss of catecholamine fluorescence from adrenergic nerves of the uterus and its arterial supply during unilateral pregnancy in the guinea-pig. The blackened area is that over which fluorescence is absent. The stage of gestation is indicated.

\section{Adrenergic fluorescence in the fetus}

The adrenergic nerves in mesenteric arteries, atrial muscle and uterine horns taken from 3 fetal guinea-pigs at 60-65 days gestation exhibited a density and intensity of fluorescence similar to that seen in adult virgin animals. 


\section{Extrauterine connections of uterine arteries and veins}

Ink perfusion of the primary uterine vein was performed in 3 animals. Passage of ink was observed from the radial veins close to their junction with the main uterine vein into a plexus of small vessels which surrounded the radial arteries (Pl. 1, Fig. 3). In transverse section these vessels were seen to be small veins which ran in the arterial adventitia. In serial sections of several radial arteries these small veins were seen to be in continuity with a system of capillaries lying in the arterial adventitia and connected to small muscular vessels which arose from the lumen of the radial artery itself (PI. 1, Figs 4 and 5).

\section{Intrauterine connections of uterine arteries and veins}

In 5 animals one uterine artery was perfused with India ink to determine whether it supplied both uterine horns. During the first few seconds of perfusion, ink appeared only in the ipsilateral horn, but subsequently it travelled in vessels lying superficially in the uterine body and appeared at the base of the contralateral horn. The ink slowly reached the middle portion of the contralateral horn, but the ovarian end of the contralateral horn remained almost unmarked.

\section{Effect of intraluminal hormones on fuorescence of uterine adrenergic axons}

In 6 animals fitted with the implants fluorescence of the intrauterine axons in the right horn was normal (PI. 2, Fig. 6) after 6-14 days but no fluorescence was observed in the left horn which contained the progesterone-impregnated pellet (PI. 2, Fig. 7). In 2 animals, only the progesteronecontaining pellet was implanted and no ligature was applied; in both fluorescence was completely absent from the implanted and contralateral horns by 10 days after implantation. In none of the 8 animals was there loss of normal fluorescence from axons in the vagina, atria or mesenteric arteries. Direct interference of progesterone with the formation of the noradrenaline fluorophore was excluded by the observation that immersion of control tissues in dimethylsulphoxide containing $10 \mathrm{mg}$ progesterone $/ \mathrm{ml}$ before freeze-drying did not affect normal development of axonal fluorescence. The rate of hormone absorption during implantation, as judged by changes in pellet weight after brief drying in a warming oven, appeared rather variable but in all instances was less than $0.5 \mathrm{mg} /$ day. Implantation into one uterine horn of pellets containing $40 \mathrm{mg}$ oestradiol/g for $10-15$ days had no effect on the normal pattern of fluorescence in three animals, although marked hypertrophy of the uterus and mammary tissue indicated that absorption had occurred.

In 4 animals, subcutaneous implantation on the shoulder of $100 \mathrm{mg}$ fused progesterone pellets (Organon) for 14 days (rate of absorption $0.5 \mathrm{mg} /$ day) had no effect on uterine fluorescence. Daily intramuscular injection of $0.5 \mathrm{mg}$ progesterone (Schering) for 7 days similarly had no effect on uterine fluorescence in another 4 animals.

\section{Effect of implanted progesterone on fuorescence of adrenergic vasomotor axons in skeletal muscle}

Five virgin guinea-pigs were given implants in the gastrocnemius muscles to determine whether the local loss of fluorescence seen during progesterone implantation in the uterine horn was specific for that organ. The animals were killed 8-33 days later. In all cases arterioles running through the gastrocnemius in the vicinity of the pure wax pellet were accompanied by a plexus of brightly fluorescent adrenergic axons (PI. 2, Fig. 8). By contrast, the arterioles which lay close to the progesterone-containing pellets were completely devoid of fluorescent axons in 4 out of the 5 animals (Pl. 2 , Fig. 9). In 4 other animals, pellets containing $40 \mathrm{mg}$ oestradiol $/ \mathrm{g}$ had no effect on the vasomotor fluorescence of arterioles close to the site of implantation in the gastrocnemius muscle. 


\section{Discussion}

Sjöberg (1968) observed that hypogastric nerve section below the inferior mesenteric ganglion in the guinea-pig did not cause degeneration of the intrauterine adrenergic axons. He concluded that these axons arose from 'short' adrenergic neurones which had their cell bodies in the pelvic plexus. Other workers have considered that the adrenergic supply to the guinea-pig uterus is by 'long' neurones which travel with the ovarian artery (Isaac et al., 1969; Kulkarni et al., 1976). The present results confirm the report of Langley \& Anderson (1895) that the guinea-pig uterus receives sympathetic axons from the ovarian and pelvic ends and offer a way of reconciling the conflicting views held by later workers. While the ovarian end of the uterine horn is supplied almost entirely by the ovarian nerves, the cervical portion of the horn is supplied almost entirely by nerves travelling from the pelvic area. The central part of the horn receives nerves from both sources. This means that, depending on the region of the uterus examined and probably also to some extent on variability between animals, section of the ovarian or the hypogastric supply could be interpreted as causing complete or no denervation.

In the rabbit, Owman \& Sjöberg (1966) reported the existence of adrenergic ganglion cells in the utero-vaginal wall and concluded that these were the cell bodies of the uterine adrenergic supply. We have been unable to find an equivalent population of ganglion cells in the wall of the uterine body or the vagina of the guinea-pig and have also demonstrated, by complete separation of uterine horn and body, that the adrenergic nerves to the horns do not travel from the body within the uterine wall. By contrast, we did observe that extirpation of the small paracervical ganglia, which lie close to the uterine artery and which supply the cell bodies of the cholinergic vasodilator fibres to this vessel (Bell, 1973), produced degeneration of adrenergic axons supplying both the myometrium and the blood vessels in the cervical part of the uterine horn. Some of these axons probably originated from the small population of paracervical ganglion cell bodies which exhibited catecholamine fluorescence. However, destruction of the paracervical ganglia would also have caused degeneration of any postganglionic axons of sympathetic chain origin which were travelling to the uterine artery through this area.

We observed that the loss of fluorescence from intrauterine adrenergic axons during pregnancy was initially restricted to that area of the uterine wall immediately adjacent to the site of implantation. With advancing gestation the area affected spread radially outwards to include both the entire pregnant horn and, later, the empty horn as well. This sequence of events suggests that the factor responsible for loss of fluorescence originates from the fetus or placenta, and gains access to the intrauterine adrenergic axons not via the systemic circulation but by passage through the uterine tissue. We observed that in female fetuses near to term the fluorescence of adrenergic nerves, in the uterus and elsewhere, was similar to that observed in normal non-pregnant adult animals. As there is no reason to suspect that fetal nerves are resistant to the hormonally-induced loss of fluorescence, this suggests that the placenta, rather than the fetus, is the site of origin of the factor involved.

The precise pathway by which this factor reaches those parts of the uterus remote from the implantation site is not clear. We demonstrated anatomical continuity between the radial veins and radial arteries, but in the absence of information on relative pressures in the two vessels the significance of these connections is uncertain. Despite their localization to the arterial adventitia and the small size of the parent vessels concerned it is possible that they represent a vasa vasorum, functional over the period of pregnancy when hypertrophy of the radial arteries occurs. We also demonstrated anastomotic vascular connections by which material could pass across the uterine body from one horn to the other. However, as Egund \& Carter (1974) have reported that no such anastomoses are detectable in the intact animal they may not be functionally patent.

Implantation into the lumen of one uterine horn of pellets containing progesterone produced disappearance of fluorescent nerves within the uterus similar to that seen during pregnancy. If no obstruction was placed between the two horns then both exhibited loss of fluorescence, but if the progesterone-containing horn was ligated at its base then the effect was restricted to that horn. No diminution of fluorescence was seen following intraluminal implantation of pellets containing oestradiol or composed of wax alone. These results suggest that progesterone is the placental factor 
responsible for intrauterine diminution of fluorescence during pregnancy; the time at which this diminution is first noted (about 20 days of gestation) corresponds to that at which peripheral plasma progesterone concentrations rise appreciably above values in non-pregnant guinea-pigs (Challis, Heap \& Illingworth, 1971).

Pregnancy or progesterone implants caused loss of fluorescence from the 'long' adrenergic axons entering the uterus with the ovarian artery as well as from the 'short' pelvic axons and progesterone implants in skeletal muscle also caused loss of fiuorescence from adjacent vasomotor axons. The restriction of the loss during pregnancy to the intrauterine axons may therefore not be related to any peculiarity of these axons but to the fact that the relatively high concentration of progesterone required to produce the effect is not attained in the systemic circulation.

The mechanism by which progesterone might affect catecholamine fiuorescence is unknown, but the observation that uterine noradrenaline content is not markedly depressed during pregnancy suggests that changes in binding of the releasable amine rather than the total axonal amine may be involved (Bell, 1974).

This work was supported by a continuing grant from the National Heart Foundation of Australia and by equipment grants from the Australian Research Grants Committee and the University of Melbourne. S.J.M. received financial assistance as a B. Med. Sci. candidate from the National Health and Medical Research Council. Organon (Aust.) generously supplied fused progesterone pellets and Schering Pty. Ltd donated injectable progesterone (Proluton). We should like to thank Dr Marthe Vogt for helpful discussions.

\section{References}

BeLl, C. (1972) Autonomic nervous control of reproduction: circulatory and other factors. Pharmac. Rev. 24, 657-736.

BeLL, C. (1973) Selective cholinergic denervation of the uterine artery in the guinea-pig. Experientia 30, 257258.

BELL, C. (1974) Control of uterine blood flow in pregnancy. Med. Biol. 52, 219-228.

Challis, J.K.G., Heap, R.B. \& Illingworth, C.D. (1971) Concentrations of oestrogens and progesterone in the plasma of non-pregnant, pregnant and lactating guinea-pigs. $J$. Endocr. 51, 333-345.

DRAPER, R.L. (1920) The prenatal growth of the guineapig. Anat. Rec. 18, 369-392.

EgUND, N. \& CARTER, A.M. (1974) Uterine and placental circulation in the guinea-pig: an angiographic study. J. Reprod. Fert. 40, 401-410.

Falck, B. \& OWman, C. (1965) A detailed methodological description of the fluorescence method for the cellular demonstration of biogenic monoamines. Acta Univ. lund. (II) 7, 1-23.

IsaAC, P.F., Pennefather, J.N. \& Silva, D.G. (1969) The ovarian and hypogastric innervation of the guinea-pig uterus. Eur.J. Pharmac. 5, 384-390.

KUlKaRNi, P.S., WaKade, A.R. \& KiRPEKAR, S.M. (1976) Sympathetic innervation of guinea-pig uterus and ovary. Am. J. Physiol. 230, 1400-1405.

Langley, J.N. \& Anderson, H.K. (1895) The innervation of the pelvic and adjoining viscera. J. Physiol., Lond. 19, 71-138.
McEwan, L.M. (1956) Effect on the isolated rabbit heart of vagal stimulation and its modification by cocaine, hexamethonium and ouabain. J. Physiol., Lond. 131, 678-689.

Nakanishi, H., Mclean, J., Wood, C. \& Burnstock, G. (1968) The role of sympathetic nerves in control of the nonpregnant and pregnant human uterus. $J$. Reprod. Med. 2, 20-33.

OWman, C. \& Sjöberg, N.-O. (1966) Adrenergic nerves in the female genital tract of the rabbit. With remarks on cholinesterase-containing structures. Z. Zellforsch. mikrosk. Anat. 74, 182-197.

Owman, C., SuöberG, N.-O. \& Suöstrand, N.-O. (1974) Short adrenergic neurones, a peripheral neuroendocrine mechanism. In Amine Fluorescence Histochemistry, pp. 47-66. Eds M. Fujiwara, \& C. Tanaka. Igaku Shoin Ltd, Tokyo.

Rosengren, E. \& SjöberG, N.-O. (1968) Changes in the amount of adrenergic transmitter in female genital tract of rabbit during pregnancy. Acta physiol. scand. 72, 412-424.

Ryan, M.J., Clark, K.E. \& Brody, M.J. (1974) Neurogenic and mechanical control of canine uterine vascular resistance. Am. J. Physiol. 227, 547-555.

SıöbERG, N.-O. (1968) Consideration on the cause of disappearance of the adrenergic transmitter in uterine nerves during pregnancy. Acta physiol. scand. 72 , $510-517$.

SuöstRAND, N.-O. (1965) The adrenergic innervation of the vas deferens and the accessory male genital glands. Acta physiol. scand. 65, Suppl. 257. 


\section{EXPLANATION OF PLATES}

\section{PLATE 1}

The horizontal bar in Fig. 2 represents $100 \mu \mathrm{m}$ for Figs 1, 2, 4 and 5. The vertical bar in Fig. 3 represents $1 \mathrm{~mm}$ for that figure.

Fig. 1. Section from the middle of the uterine horn of a virgin guinea-pig, showing catecholamine fiuorescence of the adrenergic axons associated with myometrium and with intrauterine arteries.

Fig. 2. Section from the equivalent portion of the non-pregnant horn in a unilaterally pregnant guinea-pig (gestational age 60 days). Note the absence of fluorescence around the arteries (arrows) and in the myometrium.

Fig. 3. Demonstration by ink perfusion through the uterine venous supply (V) of a venous plexus which arises from the radial vein $(\mathrm{X})$ and ramifies over the accompanying radial artery (A).

Figs 4 and 5. Haematoxylin-eosin-stained transverse sections from the radial artery and vein shown in Fig. 3. The plane of section in Fig. 4 is through the point of branching of the radial vein (X). The resultant venous plexus surrounding the artery can be seen as ink-containing profiles localized to the arterial adventitia (arrows). Fig. 5 is a section approximately $2 \mathrm{~mm}$ distal to that in Fig. 4 and shows a sidebranch of the radial artery entering the arterial adventitia (arrow). Examination of serial sections between the two points confirmed that the adventitial artery and veins were in continuity.

\section{PLATE 2}

The horizontal bar in Fig. 7 represents $100 \mu \mathrm{m}$ for all Figures.

Fig. 6. Intense fluorescence of adrenergic axons associated with myometrium and intrauterine arteries from a uterine horn into which a $50 \mathrm{mg}$ wax pellet had been implanted 7 days before.

Fig. 7. Absence of fluorescence from myometrium and from arteries (arrows) in the contralateral horn implanted, 7 days before, with a $50 \mathrm{mg}$ wax pellet containing $400 \mathrm{mg}$ progesterone/g. Transfer of progesterone into the control horn was prevented by ligating the treated horn below the implant.

Fig. 8. Intense fluorescence of adrenergic axons associated with arterioles in the gastrocnemius muscle, close to the site of implantation of a $20 \mathrm{mg}$ wax pellet 13 days before.

Fig. 9. Absence of fluorescence from arterioles (arrows) in the contralateral gastrocnemius muscle close to the site of implantation, 13 days before, of a $20 \mathrm{mg}$ wax pellet containing $400 \mathrm{mg}$ progesterone/g. 
PLATE: 1
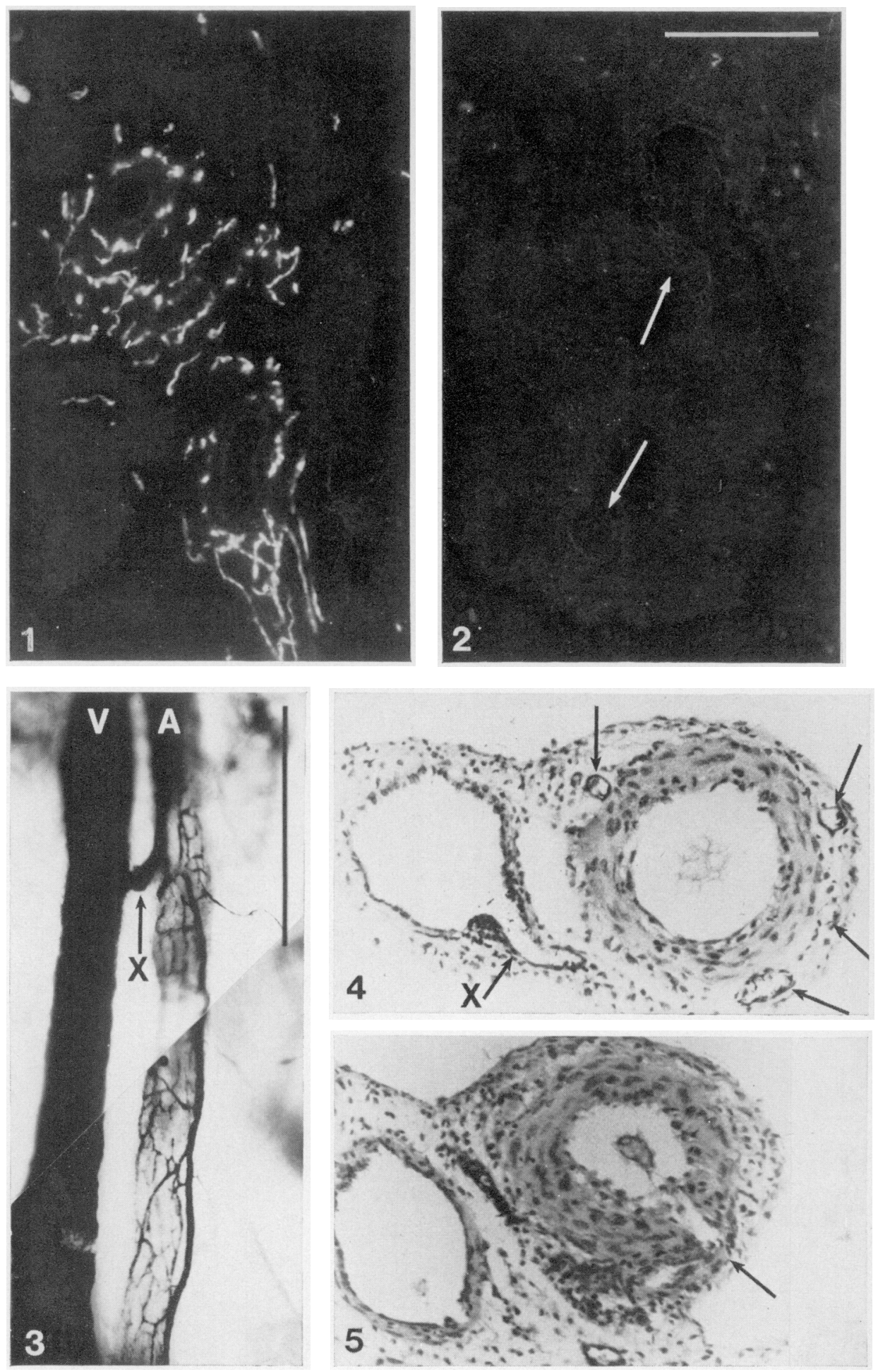

(Facing P. 58) 
$\because 112$
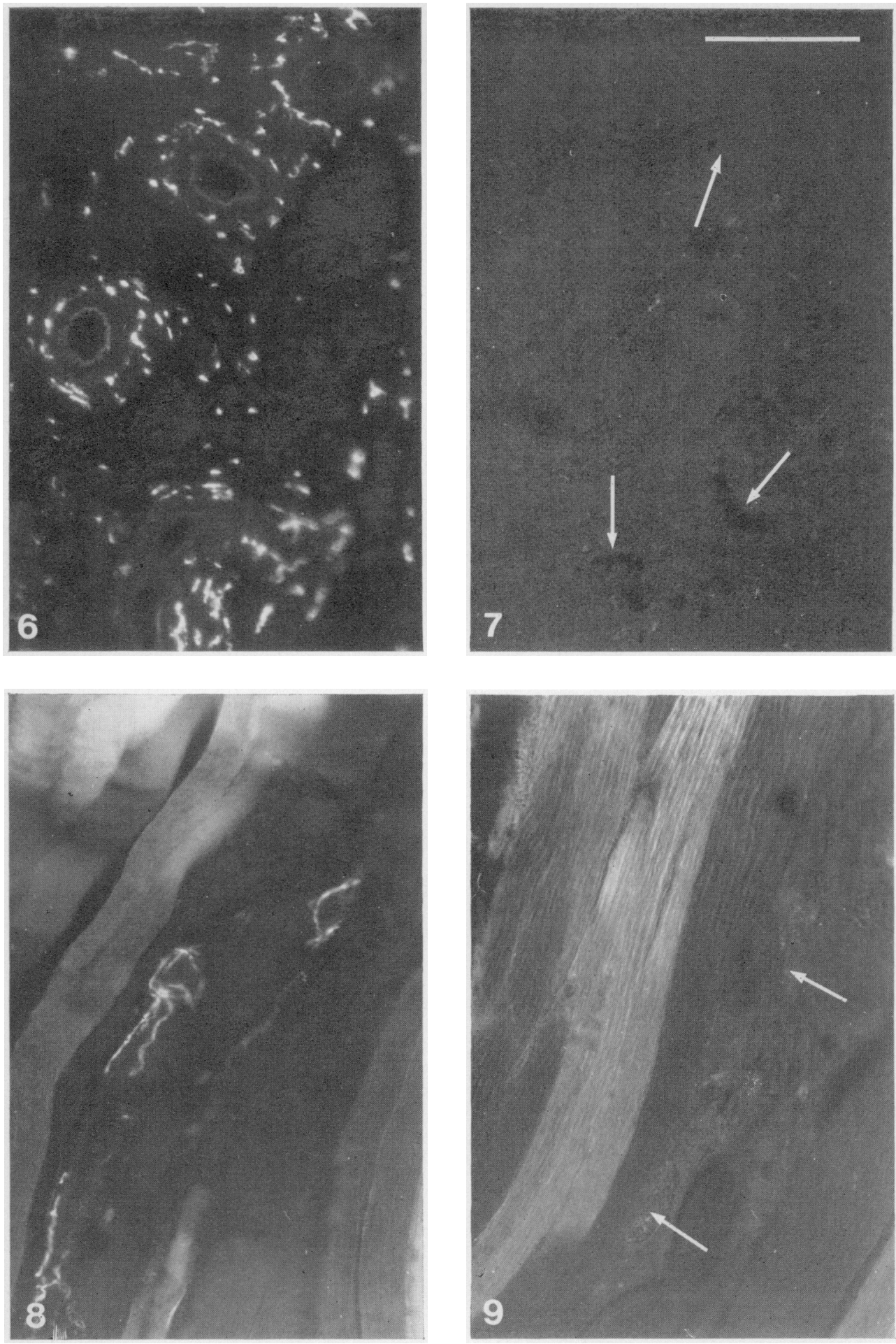